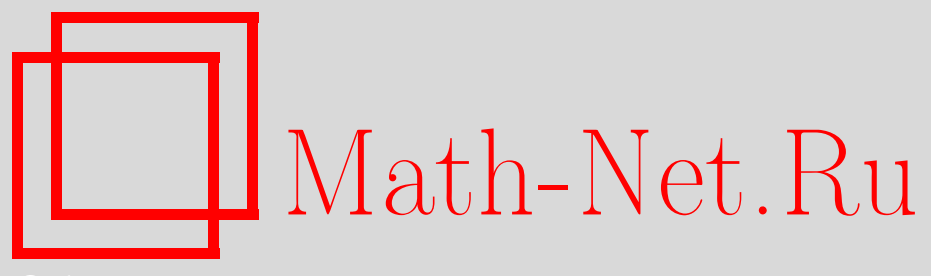

Н. Б. Енгибарян, О факторизации интегральных операторов в пространствах суммируемых функций, Изв. РАН. Сер. матем., 2009, том 73, выпуск 5, 67-82

DOI: https://doi.org/10.4213/im2713

Использование Общероссийского математического портала Math-Net.Ru подразумевает, что вы прочитали и согласны с пользовательским соглашением http://www.mathnet.ru/rus/agreement

Параметры загрузки:

IP: 54.224 .60 .19

26 апреля 2023 г., $11: 12: 17$

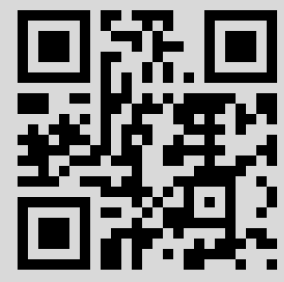


УДК 517.9

\author{
Н. Б. Енгибарян
}

\title{
О факторизации интегральных операторов в пространствах суммируемых функций
}

Рассмотрена факторизация $I-K=\left(I-U^{+}\right)\left(I-U^{-}\right)$, где $I-$ единичный оператор, $K$ - интегральный оператор, действующий в некотором банаховом пространстве функций, суммируемых по мере $\mu$ на $(a, b) \subset(-\infty,+\infty)$, непрерывной относительно меры Лебега:

$$
(K f)(x)=\int_{a}^{b} k(x, t) f(t) \mu(d t), \quad x \in(a, b),
$$

а $U^{ \pm}$- искомые вольтерровы операторы. Получено необходимое и достаточное условие существования факторизации для достаточно широкого класса операторов $K$ с положительными ядрами и для операторов Гильберта-Шмидта.

Библиография: 10 наименований.

Ключевые слова: функции, суммируемые по мере, интегральные операторы, вольтеррова факторизация.

\section{$\S 1$. Введение}

Пусть $K$ - интегральный оператор, действующий в некотором банаховом пространстве $E$ функций, суммируемых по мере $\mu$ на $(a, b) \subset(-\infty, \infty)$ :

$$
(K f)(x)=\int_{a}^{b} k(x, t) f(t) \mu(d t), \quad x \in(a, b) .
$$

Рассмотрим задачу факторизации:

$$
I-K=\left(I-U^{+}\right)\left(I-U^{-}\right),
$$

где $I$ - единичный оператор, а $U^{-}$и $U^{+}-$искомые формально вольтерровы операторы, действующие в $E$ :

$$
\begin{array}{ll}
\left(U^{+} f\right)(x)=\int_{a}^{x} u^{+}(x, t) f(t) \mu(d t), & x \in(a, b), \\
\left(U^{-} f\right)(x)=\int_{x}^{b} u^{-}(x, t) f(t) \mu(d t), & x \in(a, b) .
\end{array}
$$

В классической теории вольтерровой факторизации интегральных операторов (см. [1]) изучена задача факторизации обратного оператора:

$$
(I-K)^{-1}=\left(I+V^{-}\right)\left(I+V^{+}\right),
$$

(C) Н. Б. ЕнгиьАрян, 2009 
в пространстве $L_{2}(a, b),-\infty<a<b<+\infty$, где $K$ - интегральный оператор Гильберта-Шмидта (оператор с квадрируемым ядром):

$$
\begin{gathered}
(K f)(x)=\int_{a}^{b} k(x, t) f(t) d t, \quad x \in(a, b), \quad-\infty<a<b<+\infty, \\
\iint k^{2}(x, t) d x d t<+\infty .
\end{gathered}
$$

Центральным результатом является следующая теорема Гохберга-Крейна.

ТЕОРема ГК. Пусть скалярный или матричный интегралъный оператор имеет вид (1.5). Для существования факторизации (1.4), где $V^{ \pm}$- вольтерровы операторы с квадрируемыми ядрами, необходимо и достаточно, чтобы при любом $s \in(a, b]$ уравнение $f(x)=g(x)+\int_{a}^{s} k(x, t) f(t) d t, x \in(a, s)$, было разрешимо в пространстве $L_{2}(a, s)$.

Самостоятельно развивалась факторизационная теория уравнений ВинераХопфа второго рода и смежных классов уравнений типа свертки.

В работах [2]-[5] с различной степенью общности возникли нелинейные уравнения факторизации (НУФ) для линейных интегральных и более общих операторов. Математическая теория НУФ развита автором и его соавторами (см. [5]-[9] и др.). Получены результаты по факторизации обратимых и необратимых, скалярных и векторных операторов в банаховых пространствах, а также по соответствующим интегральным и дискретным уравнениям. Наиболее полные результаты относятся к случаю, когда ядро положительно:

$$
k \geqslant 0 \text {, }
$$

в диссипативном (докритическом) и консервативном (критическом) случаях, представляющих интерес в математической физике и в теории вероятностей.

Возможности использования НУФ при изучении экзистенциальных вопросов в случае, когда условие положительности (1.6) не выполняется, остались в недостаточной степени раскрытыми.

В настоящей работе рассматриваются вопросы из теории вольтерровых интегральных операторов в банаховых пространствах суммируемых функций. Рассматриваются интегральные операторы вида (1.1), (1.3), где $\mu$ - мера на промежутке $J_{0}=(a, b) \subset(-\infty,+\infty)$, непрерывная относительно меры Лебега:

$$
m(S)=0 \quad \Longrightarrow \quad \mu(S)=0, \quad S \subset(a, b) .
$$

Развивается процедура рекуррентного характера построения факторизации (1.2). Получены следующие основные результаты:

а) необходимое и достаточное условие существования факторизации (1.2) в случае широкого класса операторов с положительными ядрами (этот результат является новым также в случае меры Лебега на конечном промежутке);

б) обобщение теоремы ГК на случай операторов Гильберта-Шмидта вида (1.1).

Метод НУФ сочетается с факторизацией вдоль цепочки проекторов. Такая факторизация была использована в [1]. Результаты работы легко переносятся на случай интегральных операторов, действующих в пространствах конечномерных вектор-функций. 


\section{§ 2. Классы интегральных операторов}

2.1. Идеальные пространства суммируемых функций. Пусть $\mu$ - мера на промежутке $J_{0}=(a, b) \subset(-\infty,+\infty)$, непрерывная относительно меры Лебега (см. (1.7)). Обозначим через $E=E(\mu)$ некоторое идеальное банахово пространство вещественных функций, измеримых по мере $\mu$. Условие идеальности означает, что если $f \in E$ и $|\varphi(t)| \leqslant|f(t)|$ (п. в. по $\mu$ ), то $\varphi \in E$ и $\|\varphi\|_{E} \leqslant\|f\|_{E}$.

В качестве $E$ можно взять пространство $L_{p}(\mu), 1 \leqslant p<+\infty$, с нормой $\|f\|_{p}=\left(\int|f(x)|^{p} \mu(d x)\right)^{\frac{1}{p}}$ (в том числе различные весовые пространства), а также пространства Орлича, Марцинкевича, Лоренца.

2.2. Проекторы в $E$. Пусть $p_{s}, s \in(a, b) \subset(-\infty,+\infty),-$ параметризованное семейство проекторов, действующих в $E$. Если $s<b$, то $p_{s}$ определяется следующим образом:

$$
\left(p_{s} f\right)(x)=\kappa_{s}(x) f(x), \quad f \in E, \quad s \in \bar{J}=[a, b],
$$

где $\kappa_{s}$ - характеристическая функция интервала $(a, s)$. По определению имеем $p_{a}=O, p_{b}=I$, где $O$ - нулевой оператор, а $I$ - единичный оператор в $E$.

Отметим, что открытость или замкнутость исходного промежутка $J_{0}$ несущественна, а в определении проекторов $p_{s}$ собственные или несобственные точки $a$ и $b$ играют существенную роль.

Из идеальности $E$ следует, что $p_{s}$ является линейным ограниченным оператором в $E$, причем $\left\|p_{s}\right\| \leqslant 1$, т. е.

$$
\left\|p_{s} f\right\|_{E} \leqslant\|f\|, \quad f \in E .
$$

Проектор $p_{s}$ переводит банахово пространство $E$ в его подпространство $E_{s}=$ $p_{s}(E) \subset E$ функций таких, что $f(x)=0, x \geqslant s$. Имеем $E_{b}=E$, а пространство $E_{a}$ состоит только из нулевого элемента $\theta \in E$. Если $r<s$, то $E_{r} \subset E_{s}$. Все пространства $E_{s}$ идеальные.

Из перечисленных выше свойств проекторов $p_{s}$ следует, что они образуют монотонную окаймленную цепочку (см. [1]).

Пусть $p_{r s}=p_{s}-p_{r}$. Если $r \leqslant s$, то $p_{r s}$ являются проекторами.

2.3. Классы интегральных операторов. Обозначим через $\Omega$ алгебру линейных ограниченных операторов, действующих в $E$, с нормой $\|\cdot\|_{\Omega}$.

Пусть $B \subset \Omega$ - некоторая банахова алгебра с нормой $\|\cdot\|_{B} \geqslant\|\cdot\|_{\Omega}$, состоящая из регулярных интегральных операторов $K$ вида (1.1). Оператор $K$ называется регулярным, если он представляется в виде разности: $K=K_{1}-K_{2}$, где $K_{i}$, $i=1,2,-$ ограниченные интегральные операторы в $E$ с положительными ядрами $k_{i} \geqslant 0$. Введем в $B$ частичный порядок $\geqslant$; тогда запись $K_{1} \geqslant K_{2}$ означает, что $k_{1} \geqslant k_{2}$.

В качестве $B$ можно взять, например, алгебру всех регулярных интегральных операторов $K$ с нормой

$$
\|K\|_{B}=\sup _{\|f\|=1}\left\|\int_{a}^{b}|k(x, t)| f(t) \mu(d t)\right\|_{E} .
$$

Тогда $B$ является идеальным банаховым пространством.

Известен ряд критериев полной непрерывности регулярных интегральных операторов (см. [10]). 
Из теоремы Фубини следует, что в $B$ имеет место обычное правило умножения интегральных операторов. Ядром $k$ произведения $K=K_{1} K_{2} \in B$ является следующая композиция $k=k_{1} * k_{2}$ ядер сомножителей:

$$
k(x, t)=\int k_{1}(x, s) k_{2}(s, t) \mu(d s), \quad\|K\| \leqslant\left\|K_{1}\right\|\left\|K_{2}\right\| .
$$

Имеет место следующее свойство мажорантности: если $K_{2} \in B$ и $\left|k_{1}(x, t)\right| \leqslant$ $\left|k_{2}(x, t)\right|$, то выполнены включение и неравенство

$$
K_{1} \in B, \quad\left\|K_{1}\right\|_{B} \leqslant\left\|K_{2}\right\|_{B} .
$$

Важным примером пространства $B$, состоящего из вполне непрерывных операторов, служит класс $H_{2}(\mu)$ интегральных операторов Гильберта-Шмидта с нормой

$$
\|K\|_{B}^{2}=\iint k^{2}(x, t) \mu(d x) \mu(d t)<+\infty .
$$

Класс $H_{2}(\mu)$ является двусторонним идеалом в алгебре $\Omega$ линейных ограниченных операторов, действующих в $L_{2}(\mu)$.

2.4. Проекторы в $B$. Пусть $\chi_{D}$ - характеристическая функция множества $D \subset G:=(a, b) \times(a, b)=(a, b)^{2}$, измеримого по мере $\mu \times \mu$. Она определяет следующий проектор $Q_{D}$ в пространстве операторов $B$. Если $k$ - ядро оператора $K \in B$, то ядром оператора $Q_{D}(K)$ служит функция $\chi_{D}(x, y) k(x, y)$. Проектор $Q_{D}$ переводит $B$ в класс $B_{D} \subset B$ операторов, ядра которых сосредоточены в $D$.

Проекторы $Q_{D}$ являются линейными ограниченными операторами в $B$, причем $\left\|Q_{D}\right\| \leqslant 1$, т. е.

$$
\left\|Q_{D}(K)\right\|_{B} \leqslant\|K\|_{B}, \quad K \in B
$$

Произвольные два проектора $Q_{D}, Q_{S}$ коммутируют.

Ниже будут рассмотрены некоторые проекторы, связанные с областями вида

$$
G_{s}=(a, s)^{2}, \quad a<s \leqslant b, \quad G_{r s}=(r, s)^{2}, \quad S_{r s}=G_{s} / G_{r}, \quad a<r<s \leqslant b .
$$

Проектор $p_{s} \in \Omega$, введенный в п. 2.2, порождает два проектора $P_{s}, P_{s}^{\prime}$ в пространстве $B$ путем умножения операторов $K \in B$ слева и справа на $p_{s}$ : $P_{s}(K)=p_{s} K \in B, P_{s}^{\prime}(K)=K p_{s} \in B$. Проекторы $P_{s}$ и $P_{s}^{\prime}$ могут быть записаны через $Q_{D}$ следующим образом:

$$
P_{s}=Q_{D}, \quad D=(a, b) \times(a, s), \quad P_{s}^{\prime}=Q_{D}, \quad D=(a, s) \times(a, b) .
$$

Введем следующие проекторы:

$$
\begin{gathered}
P_{r s}=P_{s}-P_{r}, \quad P_{r s}^{\prime}=P_{s}^{\prime}-P_{r}^{\prime}, \quad s>r, \\
Q_{s}=P_{s} P_{s}^{\prime}, \quad Q_{r s}=Q_{s}-Q_{r}, \quad s>r,
\end{gathered}
$$

и классы операторов

$$
B_{s}=Q_{s}(B)=B_{G_{s}}, \quad B_{r s}=Q_{r s}(B)=B_{S_{r s}} .
$$

Если $r>s$, то по определению имеем

$$
P_{r s}=-P_{s r}, \quad P_{r s}^{\prime}=-P_{s r}^{\prime}, \quad Q_{r s}=-Q_{s r} .
$$


Мы будем рассматривать усеченные интегральные операторы

$$
K_{s}=Q_{s}(K) \in B_{s}, \quad K_{r s}=Q_{r s}(K)=K_{s}-K_{r} \in B_{r s} .
$$

Имеем

$$
\left(K_{s} f\right)(x)= \begin{cases}\int_{a}^{s} k(x, t) f(t) \mu(d t), & \text { если } x \leqslant s \\ 0, & \text { если } x>s .\end{cases}
$$

Если $K$ вполне непрерывный, то операторы $K_{r s}$ также вполне непрерывны.

Для произвольных $K, T \in B$ имеют место равенства

$$
P_{r s}(K T)=\left(P_{r s}(K)\right) T, \quad P_{r s}^{\prime}(K T)=K\left(P_{r s}^{\prime}(T)\right) .
$$

Приведем одно легко проверяемое алгебраическое свойство подклассов из $B$.

Лемма 2.1. Пусть $K_{r} \in B_{r}, T_{r s} \in B_{r s}, r<s$. Тогда

$$
K_{r} T_{r s} \in B_{(a, r) \times(r, s)} \subset B_{r s}, \quad T_{r s} K_{r} \in B_{(r, s) \times(a, r)} \subset B_{r s} .
$$

2.5. Локально сжимающие операторы. Оператор $K \in B$ назовем локально q-ограниченным вдоль иепочки $\left\{Q_{a}\right\}$, если каждая точка $r \in[a, b]$ обладает такой окрестностью $\tau_{r}$, что

$$
\left\|Q_{s l}(K)\right\|_{B} \leqslant q, \quad s, l \in \tau_{r} .
$$

В случае $q<1$ оператор $K$ назовем локально сжимающим.

Лемма 2.2. Пусть оператор $K \in B$ локально q-ограниченный. Тогда существует конечное разбиение

$$
\Pi[a, b]=\left\{s_{k}\right\}, \quad a=s_{0}<s_{1}<\cdots<s_{n}=b,
$$

компакта $[a, b]$ такое, что

$$
\left\|K_{s_{i}, s_{i+1}}\right\|=\left\|Q_{s_{i}, s_{i+1}}(K)\right\|_{B} \leqslant q, \quad i=0, \ldots, n-1 .
$$

ДоКАЗАТЕЛЬСтво. Из условия леммы следует, что существует открытое покрытие $\tilde{\tau}=\left\{\tau_{r}\right\}$ компакта $[a, b]$ (состоящее из открытых интервалов и крайних полуинтервалов вида $[a, \alpha),(\beta, b])$ такое, что выполняются условия $(2.12)$. Согласно лемме Гейне-Бореля из этого покрытия можно выделить конечное подпокрытие $\tau=\left\{\tau_{r_{j}}\right\}, j=1, \ldots, m$. Обозначим через $\eta_{0} \in \tau$ ту окрестность точки $a=s_{0}$, которая имеет наибольший правый конец $\alpha_{1}$. Далее выберем ту окрестность (или одну из тех окрестностей) $\eta_{1} \in \tau$ точки $\alpha_{1}$, которая имеет наибольший правый конец $\alpha_{2}$. Продолжим этот процесс. На $i$-м шаге выберем ту окрестность $\eta_{i} \in \tau$ уже выбранной точки $\alpha_{i-1}$, которая имеет наибольший правый конец $\alpha_{i}$. После конечного числа шагов не более $m$ мы дойдем до точки $\alpha_{n}=b$.

Определим точки разбиения $\left\{s_{i}\right\}$. В качестве $s_{i}, i<n$, можно взять произвольную точку из пересечения $\eta_{i} \cap \eta_{i+1}$. Поскольку точки $s_{i}$ и $s_{i+1}$ принадлежат одному и тому же интервалу $\eta_{i+1} \in \tau$, согласно (2.7) выполняется неравенство (2.12). Лемма доказана.

ЗАмечАниЕ 2.1. Лемма 2.2 остается в силе, если $\mu$ - произвольная $\sigma$-конечная мера на интервале $(-\infty,+\infty)$. 
ОПРеДЕЛЕНИЕ 2.1. Оператор $K \in B$ назовем непрерывным вдоль иепоч$\kappa u\left\{Q_{s}\right\}$, если оператор-функция $K_{s}=Q_{s}(K) \in B_{s}$ непрерывно (по норме) зависит от $s$ на $[a, b]$ :

$$
\left\|Q_{r s}(K)\right\|_{B} \rightarrow 0, \quad r \rightarrow s, \quad s, r \in[a, b] .
$$

Для выполнения условия (2.12) достаточно, чтобы

$$
\begin{array}{ll}
\left\|P_{r s}(K)\right\|_{B} \rightarrow 0, & r \rightarrow s, \quad s, r \in[a, b], \\
\left\|P_{r s}^{\prime}(K)\right\|_{B} \rightarrow 0, & r \rightarrow s, \quad s, r \in[a, b] .
\end{array}
$$

Обозначим через $\widetilde{B} \subset B$ класс операторов, непрерывных вдоль цепочки $\left\{Q_{s}\right\}$. Через $\breve{B} \subset \widetilde{B}$ обозначим класс операторов $K$, удовлетворяющих условиям (2.16).

Оператор $K \in \widetilde{B}$ является локально q-ограниченным для произвольного $q>0$.

Лемма 2.3. Справедливы следующие утверждения:

i) класс $\widetilde{B}$ является подпространством банахова пространства $B$;

ii) класс $\breve{B}$ является идеалом в банаховой алгебре $B$;

iii) класс $\breve{B}_{r}=Q_{r}(\breve{B})$ является идеалом в $B_{r}$.

ДокАЗАтЕльство. Докажем полноту пространства $\widetilde{B}$. Пусть последовательность $K^{(n)}$ из $\widetilde{B}$ сходится в $B: K^{(n)} \rightarrow K \in B$. Покажем, что $K \in \widetilde{B}$. С учетом (2.7) получаем $\left\|Q_{r s}(K)\right\|_{B} \leqslant\left\|Q_{r s}\left(K^{(n)}\right)\right\|_{B}+\left\|K-K^{(n)}\right\|_{B}$. Выберем номер $n$ настолько большим, чтобы выполнялось неравенство $\left\|K-K^{(n)}\right\|_{B}<\frac{\varepsilon}{2}$. В силу $K^{(n)} \in B$ можно выбрать $r$ так, чтобы $\left\|Q_{r s}\left(K^{(n)}\right)\right\|_{B}<\frac{\varepsilon}{2}$, что приводит к неравенству $\left\|Q_{r s}(K)\right\|_{B}<\varepsilon$. Утверждение і) леммы доказано. Аналогично проверяется полнота пространства $\breve{B}$. Покажем теперь, что класс $\breve{B}$ замкнут относительно умножения. Пусть $K, T \in \breve{B}$ и $W=K T$. Согласно (2.11) имеем $P_{r s}(W)=\left(P_{r s}(K)\right) T$, откуда следует $\left\|P_{r s} W\right\| \rightarrow 0$ при $r \rightarrow s$. Аналогично показывается, что $\left\|P_{r s}^{\prime} W\right\| \rightarrow 0$ при $r \rightarrow s$. Утверждение іiі) проверяется с учетом коммутативности проектора $Q_{l}$ с проекторами $P_{r s}, P_{r s}^{\prime}$. Лемма доказана.

\section{§ 3. Обратимость операторов $I-K_{r}$, резольвента Фредгольма}

3.1. Некоторые свойства резольвенты. Пусть $K \in B$. Оператор $I-K$ назовем $B$-обратимым в $E$, если существует обратный оператор

$$
(I-K)^{-1}=I+\Gamma, \quad \Gamma \in B .
$$

Оператор Г назовем резольвентой (В-резольвентой) оператора $K$.

Пусть $K_{1}, K_{2} \in B$ и операторы $I-K_{1}, I-K_{2}$ являются $B$-обратимыми: $\left(I-K_{i}\right)^{-1}=I+\Gamma_{i}, i=1,2$. Тогда таким же является произведение $I-K=$ $\left(I-K_{1}\right)\left(I-K_{2}\right)$. Резольвентой оператора $K$ является $\Gamma=\Gamma_{1}+\Gamma_{2}+\Gamma_{2} \Gamma_{1} \in B$. Если

$$
\Gamma_{1}, \Gamma_{2} \geqslant O, \quad \text { то } \quad \Gamma \geqslant O .
$$

Пусть $K \in B, q=\|K\|_{B}<1$. Тогда существует резольвента $\Gamma \in B$, которая является суммой равномерно сходящегося ряда Неймана. Если $K \geqslant O$, то $\Gamma \geqslant O$. 
Пусть $K \in \widetilde{B}$, где $\widetilde{B}$ является идеалом (хотя бы односторонним) в $\Omega$, и оператор $I-K$ обратим в $\Omega$. Покажем, что тогда

$$
(I-K)^{-1}=I+\Gamma, \quad \Gamma \in \widetilde{B} .
$$

Пусть $\widetilde{B}$ является левосторонним идеалом в $\Omega$. Обозначим $\Gamma=(I-K)^{-1} K \in \Omega$. Имеем $\Gamma=K+K \Gamma$. Из $K \in \widetilde{B}$ следует $K \Gamma \in \widetilde{B}$ и $\Gamma \in \widetilde{B}$.

Пусть $K \in B, s \in[a, b]$ и оператор $K_{s}=Q_{s}(K)$ обладает резольвентой $\Gamma_{s} \in B_{s}$. Обозначим

$$
\varphi(s)=\left\|\Gamma_{s}\right\|_{B}<+\infty .
$$

Имеем $\varphi(a)=0$. Если $K \in \widetilde{B}$, то $\left\|K_{s}\right\|_{B}$ непрерывно зависит от $s$.

ЛЕмма 3.1. Справедливы следующие утверждения:

i) пусть $K_{s} \in B_{s}$ и существует резольвента $\Gamma_{s} \in B_{s}$; тогда существует такая окрестность $\tau_{s}$ точки $s$ (по топологии в $[a, b] \subset[-\infty,+\infty]$ ), что оператор $I-K_{r}$ обратим при $r \in \tau$ :

$$
\left(I-K_{r}\right)^{-1}=I+\Gamma_{r}, \quad \Gamma_{r} \in B_{r}
$$

ii) если при этом $K \geqslant O, \Gamma_{s} \geqslant O u r>s$, mо $\Gamma_{r} \geqslant O$;

iii) функиия $\varphi(r)$, определенная по формуле (3.3) в окрестности s, непреръвна в точке $s$; если резольвента $\Gamma_{r}$ существует при произвольном $r \in[a, s]$, то функиия $\varphi$ ограничена на компакте $[a, s]$; если при этом $K \geqslant O$, то $\Gamma_{r}$ и $\varphi(r)$ монотонно зависят от $r$ :

$$
O \leqslant \Gamma_{r} \leqslant \Gamma_{s}, \quad 0 \leqslant \varphi(r) \leqslant \varphi(s), \quad r \leqslant s .
$$

ДоказАтельство. Имеет место $I-K_{r}=\left(I-K_{s}\right)\left(I-T_{r s}\right)$, где $T_{r s}=$ $\left(I+\Gamma_{s}\right)\left(K_{r}-K_{s}\right)$. Согласно утверждению ii) леммы 2.3 имеем $T_{r s} \in \widetilde{B}_{r s}$. Согласно определению класса $\widetilde{B}$ существует такая окрестность $\tau_{s}$ точки $s$, что $\left\|K_{r}-K_{s}\right\| \leqslant\left(1+\left\|\Gamma_{s}\right\|\right)^{-1} q, q<1$, при $r \in \tau_{s}$. Тогда $\left\|T_{r s}\right\| \leqslant q<1$ и оператор $I-T_{r s}$ будет $\widetilde{B}$-обратимым. Поэтому

$$
I+\Gamma_{r}=\left(I-T_{r s}\right)^{-1}\left(I+\Gamma_{s}\right), \quad \Gamma_{r} \in \widetilde{B}_{r} .
$$

Из формулы (3.1) следует утверждение іi) леммы.

Из неравенства $\left\|\Gamma_{r}-\Gamma_{s}\right\|_{B} \leqslant\left(1+\left\|\Gamma_{s}\right\|_{B}\right)^{2}\left\|K_{r}-K_{s}\right\|_{B}$ вытекает, что $\left\|\Gamma_{r}-\Gamma_{s}\right\|_{B} \rightarrow 0$ при $r \rightarrow s$, откуда согласно $|\varphi(r)-\varphi(s)| \leqslant\left\|\Gamma_{r}-\Gamma_{s}\right\|$ следует непрерывность $\varphi$ в точке $s$. Если резольвента $\Gamma_{s}$ существует при произвольном $s \in[a, b]$, то функция $\varphi$ непрерывна на компакте $[a, b]$, стало быть, она ограничена. Лемма доказана.

3.2. Зона субкритичности, критическая точка. Пусть $K \in \widetilde{B}$. Обозначим через $J=J(K) \subset[a, b]$ следующее множество:

$$
s \in J, \quad \text { если } \forall r \in[a, s] \exists\left(I-K_{r}\right)^{-1}=I+\Gamma_{r}, \quad \Gamma_{r} \in \widetilde{B}_{r} .
$$

Множество $J$ не пусто. Поскольку $K_{a}=O$, то $\Gamma_{a}=O$, т. е. резольвента $\Gamma_{r}$ существует при $r=a$, и, тем самым, согласно лемме 3.1 она существует в некоторой окрестности точки $a$.

Обозначим $\beta=\sup J \leqslant b$. Из определения множества $J$ и леммы 3.1 следует, что если $\beta<b$, то $J=[a, \beta)$. 
Нами доказана следующая лемма.

Лемма 3.2. Справедливы утверждения:

i) если $\beta<b$, то $J=[a, \beta)$; при $\beta=b$ возможны два случая: $J=[a, b) u$ $J=[a, b]$

ii) пусть $s \in J$; тогда функиия $\varphi(r)=\left\|\Gamma_{r}\right\|_{\Omega}$ непрерьвна на отрезке $[a, s] u$ имеют место неравенства (3.4).

В случае $J=[a, \beta)$ назовем интервал $J$ субкритической зоной, а точку $\beta \leqslant b$ назовем критической точкой. В случае $J=[a, \beta]$ критическая точка отсутствует. Тогда операторы $I-K_{r}$ являются $\widetilde{B}$-обратимыми при произвольном $r \in[a, b]$.

Отметим, что в случае оператора с положительным ядром критическая точка играет важную роль в теории цепных ядерных реакций и в некоторых других вопросах физики.

\section{§ 4. Вольтерровы операторы}

4.1. Общие свойства. Пусть $G_{r}^{ \pm} \subset G_{r}$ - следующие "треугольники":

$$
G_{r}^{+}=\left\{(x, y) \in G_{r} ; y \leqslant x\right\}, \quad G_{r}^{-}=\left\{(x, y) \in G_{r} ; y>x\right\}, \quad G^{ \pm}=G_{b}^{ \pm} \subset \mathbb{R}^{2} .
$$

Введем также "трапеции" $G_{r s}^{ \pm} \subset G_{r s}$ :

$$
G_{r s}^{+}=\left\{(x, y) \in G_{r s} ; y \leqslant x\right\}, \quad G_{r s}^{-}=\left\{(x, y) \in G_{r s} ; y>x\right\}, \quad r<s .
$$

Обозначим через $P^{+}$и $P^{-}$проекторы, действующие на операторах из $B$ посредством умножения их ядер на характеристические функции множеств $G^{+}$ и $G^{-}$соответственно. Проекторы $P^{ \pm}$переводят $\Omega$ в классы $B^{ \pm}=Q^{ \pm}(B)$ нижних и верхних формально вольтерровых (треугольных) операторов $U^{ \pm}$вида (1.3).

Классы $B^{+}$и $B^{-}$замкнуты относительно умножения и являются алгебрами, а $B$ является их прямой суммой: $B=B^{+} \oplus B^{-}$.

Проекторы $P^{ \pm}$переводят алгебру усеченных операторов $B_{r}$ в ее подалгебры $B_{r}^{ \pm}$. Имеем $B_{r}=B_{r}^{+} \oplus B_{r}^{-}$.

Оператор $U^{-} \in B^{-}$обладает цепочкой инвариантных подпространств $E_{s}=$ $p_{s}(E)$, а оператор $U^{+} \in B^{+}$обладает цепочкой $\widetilde{E}_{s}=\left(I-p_{s}\right)(E)$.

Обозначим

$$
B_{r s}^{ \pm}=B^{ \pm} \cap B_{r s}, \quad \widetilde{B}_{r s}^{ \pm}=\widetilde{B}^{ \pm} \cap \widetilde{B}_{r s}, \quad \breve{B}_{r s}^{ \pm}=\breve{B}^{ \pm} \cap \breve{B}_{r s} .
$$

Приведем некоторые алгебраические свойства классов $B_{r s}$, которые проверяются с помощью формулы композиции ядер (2.4).

ЛЕмма 4.1. Справедливы следующие утверждения:

i) классъ $B_{r s}^{ \pm}$и $\breve{B}_{r s}^{ \pm}$являются алгебрами, $B_{r s}=B_{r s}^{+} \oplus B_{r s}^{-}, \breve{B}_{r s}=\breve{B}_{r s}^{+} \oplus \breve{B}_{r s}^{-}$;

ii) nyсть $W_{r s}^{ \pm} \in B_{r s}^{ \pm} u U_{s l}^{ \pm} \in B_{s l}^{ \pm}, a \leqslant r<s<l \leqslant b$; тогда

$$
\begin{gathered}
W_{r s}^{+} U_{s l}^{+}=O, \quad U_{s l}^{-} W_{r s}^{-}=O, \\
W_{r s}^{+} W_{r s}^{-} \in B_{r s} ;
\end{gathered}
$$

ecли $W_{r s}^{ \pm} \in \widetilde{B}_{r s}^{ \pm}$, mo $W_{r s}^{+} W_{r s}^{-} \in \widetilde{B}_{r s}$.

Отметим, что класс $B_{r s}$ не замкнут относительно умножения. 
Приведем следующие важные для наших целей свойства треугольных операторов.

Лемма 4.2. Пусть $U_{s}^{ \pm} \in B_{s}^{ \pm} u r \leqslant s$. Тогда имеют место равенства

$$
\begin{gathered}
P_{r}\left(U_{s}^{+}\right)=Q_{r}\left(U_{s}^{+}\right) \in B_{r}^{+}, \quad P_{r}^{\prime}\left(U_{s}^{-}\right)=Q_{r}\left(U_{s}^{-}\right) \in B_{r}^{-}, \\
Q_{r}\left(U_{s}^{+} U_{s}^{-}\right)=Q_{r}\left(U_{s}^{+}\right) Q_{r}\left(U_{s}^{-}\right) \in B_{r} .
\end{gathered}
$$

Eсли $U_{s}^{ \pm} \in \widetilde{B}_{s}^{ \pm}$, mo $Q_{r}\left(U_{s}^{ \pm}\right) \in \widetilde{B}_{r}^{ \pm}, Q_{r}\left(U_{s}^{+} U_{s}^{-}\right) \in \widetilde{B}_{r}$

Свойства (4.4), которые мы назовем свойствами треуголъников, имеют простой геометрический смысл. Например, первое из них означает, что аннулирование ядра $u_{s}^{+}(x, t)$ при $x>r$ влечет за собой аннулирование этого ядра при $t>r$. Свойства (4.5) следуют из (4.4) согласно (2.11).

4.2. Резольвенты вольтерровых операторов. Пусть $U^{ \pm} \in B^{ \pm}$. Операторы $I-U^{ \pm}$назовем $B^{ \pm}$-обратимыми (или просто обратимыми), если

$$
\left(I-U^{ \pm}\right)^{-1}=I+V^{ \pm}, \quad V^{ \pm} \in B^{ \pm} .
$$

Если $\left\|U^{ \pm}\right\|_{B}<1$, то операторы $I-U^{ \pm}$обратимы.

Из равенств (4.4) и (2.15) следует, что если $U^{ \pm} \in \widetilde{B}^{ \pm}$, то $V^{ \pm} \in \widetilde{B}^{ \pm}$.

Умножая равенства $V^{+}=U^{+}+U^{+} V^{+}$и $V^{+}=U^{+}+V^{+} U^{+}$слева на $p_{s}$, с учетом равенств (2.11) получаем $V_{s}^{+}=U_{s}^{+}+U_{s}^{+} V_{s}^{+}$и $V_{s}^{+}=U_{s}^{+}+V_{s}^{+} U_{s}^{+}$, где $U_{s}^{+}=Q_{s}\left(U^{+}\right), V_{s}^{+}=Q_{s}\left(V^{+}\right)$. Поэтому $V_{s}^{+}$является резольвентой оператора $U_{s}^{+}$. Ясно, что в качестве исходного оператора $U^{+}$можно взять $U_{l}^{+}=Q_{l}\left(U^{+}\right), l>s$. Поэтому из $B^{+}$-обратимости оператора $I-U_{l}^{+}$следует обратимость $I-U_{s}^{+}, s<l$. Аналогично, из $B^{-}$-обратимости оператора $I-U_{l}^{-}$ следует обратимость $I-U_{s}^{+}, s<l$. Имеют место формулы

$$
V_{s}^{ \pm}=Q_{s}\left(V_{l}^{ \pm}\right), \quad s<l .
$$

Из (4.7) следует, что если $U^{ \pm} \in B_{s}^{ \pm}$, то $V^{ \pm} \in B_{s}^{ \pm}$.

Хорошо известным примером оператора $U^{+} \in B^{+}$, не имеющего резольвенту в $B^{+}$, является $U^{+} f(x)=\frac{1}{x} \int_{0}^{x} f(t) d t$, действующий в пространствах $L_{p}(0, b)$, $b<+\infty$. Этот оператор обладает неподвижными векторами $\varphi(t)=$ const.

Согласно (4.4) операторы $U^{+} \in B^{+}$и $U^{-} \in B^{-}$являются локально $q$-сжимающими, если в некоторой окрестности $\tau_{r}$ каждой точки $r$ соответственно выполняются неравенства

$$
\left\|P_{s l}\left(U^{+}\right)\right\| \leqslant q<1, \quad\left\|P_{s l}^{\prime}\left(U^{-}\right)\right\| \leqslant q<1, \quad l \in \tau_{r} .
$$

Операторы из $\widetilde{B}^{ \pm}$являются локально сжимающими с произвольным коэффициентом сжатия $q \in(0,1)$.

Лемма 4.3. Справедливы следующие утверждения:

i) пусть $U^{+} \in B^{+}, U_{r s}^{+}=p_{r s} U^{+}=U_{s}^{+}-U_{r}^{+}, s>r$; тогда имеет место разложение

$$
I-U_{r l}^{ \pm}=\left(I-U_{r s}^{+}\right)\left(I-U_{s l}^{+}\right), \quad r<s<l ;
$$

ii) пусть $\Pi[a, b]$ - конечное разбиение вида (2.13) и $\Delta_{k}=U_{s_{k}+1}^{+}-U_{s_{k}}^{+}=$ $U_{s_{k}, s_{k}+1}^{+} \in B_{s_{k}, s_{k}+1}^{+} ;$имеет место разложение

$$
I-U^{+}=\left(I-\Delta_{1}\right) \cdots\left(I-\Delta_{n}\right)
$$


iii) nусть оператор $U^{+} \in B^{+}$обладает $B^{+}$-резолъвентой $V^{+}$; тогда при произвольном $s \in[a, b]$ резольвентой оператора $U_{s}^{+}=p_{s} U^{+}$служит $V_{s}^{+}=$ $p_{s} V^{+} \in B_{s}^{+} ;$если $V^{+} \geqslant O$, mо $V_{s}^{+} \geqslant O$;

iv) произвольный оператор $U^{+} \in \widetilde{B}^{+}$обладает резолъвентой $V^{+} \in \widetilde{B}^{+}$, причем если $U^{+} \geqslant O$, то $V^{+} \geqslant O$;

v) аналогичные утверждения имеют место для операторов $U^{-} \in B^{-}$.

ДокАзАтельство. Разложение (4.9) следует из равенства (4.1). Разложение (4.10) следует из (4.9). Утверждение iii) доказано выше (см. формулу (4.7)). Докажем теперь утверждение iv). Из $U^{+} \in \widetilde{B}^{+}$следует, что оператор $U^{+}$локально сжимающий. Согласно лемме 2.2 существует такое конечное разбиение $\Pi[a, b]$, что $\left\|\Delta_{k}\right\|_{B}<1, k=1, \ldots, n$. Тогда в разложении (4.10) все сомножители будут обратимыми: $\left(I-\Delta_{k}\right)^{-1}=I+\rho_{k}, \rho_{k} \in B_{s_{k}, s_{k}+1}^{+}$. Поэтому $\left(I-U^{+}\right)^{-1}=I+V^{+}, V^{+}=\rho_{1}+\cdots+\rho_{n}+\cdots \in B^{+}$. Если $U^{+} \geqslant O$, то $\Delta_{k} \geqslant O$, $\rho_{k} \geqslant O, V^{+} \geqslant O$. Лемма доказана.

\section{§ 5. Задача факторизации}

5.1. Нелинейное уравнение факторизации. Рассмотрим задачу (1.2), где $K \in B$ - оператор вида (1.1), а $U^{ \pm} \in B^{ \pm}$- искомые вольтерровы операторы вида (1.3). Факторизацию (1.2) назовем канонической, если операторы $I-U^{ \pm}$ являются $B^{ \pm}$-обратимыми. Тогда $I-K$ будет $B$-обратимым. Легко убедиться в единственности канонической факторизации. Из п. iv) леммы 4.3 следует, что в классах $\widetilde{B}$ факторизация всегда каноническая.

Приведем нелинейные уравнения факторизации (НУФ). Равенство (1.2) эквивалентно равенству

$$
U^{+}+U^{-}=K+U^{+} U^{-}
$$

Применяя к обеим частям (5.1) проекторы $P^{ \pm}$, приходим к НУФ в операторной форме:

$$
U^{+}=K^{+}+P^{+}\left(U^{+} U^{-}\right), \quad U^{-}=K^{-}+P^{-}\left(U^{+} U^{-}\right),
$$

где $K^{ \pm}=P^{ \pm}(K)$.

Обратно, пусть $U^{ \pm} \in \Omega^{ \pm}$удовлетворяют уравнениям (5.2). Складывая эти соотношения, получаем равенство (5.1).

Итак, факторизация (1.2) эквивалентна системе уравнений (5.2) относительно $U^{ \pm}$, называемых нелинейными уравнениями факторизации.

Запишем НУФ (5.2) в раскрытой форме, через ядра. С учетом правила композиции ядер (2.4) получаем

$$
\begin{array}{ll}
u^{+}(x, y)=k(x, y)+\int_{a}^{y} u^{+}(x, t) u^{-}(t, y) \mu(d t), & x>y, \\
u^{-}(x, y)=k(x, y)+\int_{a}^{x} u^{+}(x, t) u^{-}(t, y) \mu(d t), & x<y .
\end{array}
$$

5.2. Инвариантное погружение. Нелинейные уравнения факторизации (5.2) и (5.3) обладают рядом замечательных свойств. Следующая лемма выражает их вольтерров характер.

В уравнениях (5.3) не фигурирует верхний предел интегрирования $b$ в явном виде. Поэтому, аналогично задаче Коши для дифференциальных уравнений 
или уравнениям типа Вольтерра, можно заранее не фиксировать ту область, в которой ищется решение НУФ. Приведем строгую формулировку.

Пусть НУФ (5.2) обладает решением $\left(U^{+}, U^{-}\right) \in B^{+} \times B^{-}$и $s \in(a, b]$. Применяя к равенству (5.1) проектор $Q$, с учетом (4.5) получаем равенство $U_{s}^{+}+U_{s}^{-}=K_{s}+U_{s}^{+} U_{s}^{-}$. Это равенство эквивалентно системе (5.2), в которой оператор $K$ заменен редуцированным оператором $K_{s}=Q_{s}(K)$ :

$$
U_{s}^{ \pm}=K_{s}^{+}+P^{ \pm}\left(U_{s}^{+} U_{s}^{-}\right) .
$$

Равенство (5.4) остается в силе, если вместо исходного оператора $K$ взять оператор $K_{l}, l \geqslant s$. Поскольку $Q_{s}\left(K_{l}\right)=K_{s}$ при $s \in[a, l]$, то из разрешимости системы (5.4) при некотором значении $s=l$ следует ее разрешимость при произвольном $s \in[a, l]$, причем

$$
U_{s}^{ \pm}=Q_{s}\left(U_{l}^{ \pm}\right), \quad s \leqslant l .
$$

В случае обратимости $I-U^{ \pm}$согласно лемме 4.3 операторы $I-U_{s}^{ \pm}$обратимы, стало быть, обратимо и их произведение $I-K_{s}$. Нами доказана следующая лемма.

ЛЕмма 5.1. Справедливы следующие утверждения:

i) пусть НУФ (5.4) при некотором значении $s=l \in(a, b]$ обладает решением $\left(U_{l}^{+}, U_{l}^{-}\right) \in B_{l}^{+} \times B_{l}^{-} ;$тогда при произвольном $s \in[a, l]$ система $(5.4)$ имеет решения $U_{s}^{ \pm}=Q_{s}\left(U_{l}^{ \pm}\right)$и имеет место факторизация

$$
I-K_{s}=\left(I-U_{s}^{+}\right)\left(I-U_{s}^{-}\right), \quad U_{s}^{ \pm} \in B_{s}^{ \pm} ;
$$

ii) если факторизачия (5.6) каноническая при $s=l$, то она каноническая при произвольном $s \in[a, l]$ u оператор $I-K_{s}$ обратим; тогда операторь $Q_{s}\left(U_{l}^{ \pm}\right)$не зависят от $l$;

iii) если $K_{l} \in \widetilde{B}_{l}$, то $U_{s}^{ \pm} \in \widetilde{B}_{s}^{ \pm}$и факторизачия (5.6) каноническая;

iv) обратимость оператора $I-K_{s}$ для произвольного $s \in[a, b]$ является необходимым условием существования канонической факторизации (1.2).

5.3. Локальная разрешимость НУФ. Пусть $K \in B$. Рассмотрим семейство задач факторизации (5.6), где $K_{s}=Q_{s}(K)$, а $\left(U_{s}^{+}, U_{s}^{-}\right)$ищутся в $B_{s}^{+} \times B_{s}^{-}$. Пространство $B^{+} \times B^{-}$и его подпространства $B_{r s}^{+} \times B_{r s}^{-}$мы будем снабжать нормой $\left\|\left(U^{+}, U^{-}\right)\right\|=\max \left(\left\|U^{+}\right\|_{B},\left\|U^{-}\right\|_{B}\right)$.

Пусть $T \in B, T^{ \pm}=Q^{ \pm}(T)\left(\in B^{ \pm}\right)$. Рассмотрим вспомогательное уравнение $W^{ \pm}=T^{ \pm}+P^{ \pm}\left(W^{+} W^{-}\right)$, или уравнение в операторной форме

$$
\left(W^{+}, W^{-}\right)=H\left(W^{+}, W^{-}\right) .
$$

Оператор $H$ определяется посредством $\left(F^{+}, F^{-}\right)=H\left(W^{+}, W^{-}\right)$, где

$$
F^{ \pm}=T^{ \pm}+P^{ \pm}\left(W^{+} W^{-}\right) .
$$

Пусть $T \in B_{r s}$. Из (4.3) следует, что оператор $H$ действует в пространстве $B_{r s}^{+} \times B_{r s}^{-}$. Решение уравнения (5.7) будем искать в этом пространстве.

Обозначим через $\sigma_{\rho} \subset B_{r s}^{+} \times B_{r s}^{-}$замкнутый шар с радиусом $\rho$ и центром в нуле.

Лемма 5.2. Пусть $T \in B_{r s}, \mu:=\left\|\left(T^{+}, T^{-}\right)\right\|<\frac{1}{4} u \rho=\frac{1}{2}(1-\sqrt{1-4 \mu})$. Тогда: 
i) Н является сжимающим оператором в $\sigma_{\rho}$ с коэффиииентом сжатия $2 \rho<1$, система (5.7) имеет единственное решение $\left(W^{+}, W^{-}\right)$в $\sigma_{\rho}$, причем

$$
\left\|W^{ \pm}\right\|<\frac{1}{2}, \quad\left(I-W^{ \pm}\right)^{-1}=I+Z^{ \pm}, \quad Z^{ \pm} \in B_{r s}^{ \pm}
$$

ii) если $T^{ \pm} \geqslant O$, mo $W^{ \pm} \geqslant O, Z^{ \pm} \geqslant O$.

ДоказАТЕЛЬСтво. Из (5.8) с учетом неравенств вида $\left\|P^{ \pm}(A)\right\|_{B} \leqslant\|A\|_{B}$ (см. (2.7)) получаем $\left\|F^{ \pm}\right\| \leqslant \mu+\left\|W^{+}\right\|\left\|W^{-}\right\|$. Поэтому $\left\|\left(F^{+}, F^{-}\right)\right\| \leqslant \mu+$ $\left\|\left(W^{+}, W^{-}\right)\right\|$, откуда следует, что оператор $H$ переводит шар $\sigma_{\rho}$ в себя. Покажем, что оператор $H$ сжимающий. Пусть $\left(\widetilde{F}^{+}, \widetilde{F}^{-}\right)=H\left(\widetilde{W}^{+}, \widetilde{W}^{-}\right)$, где $\left(\widetilde{W}^{+}, \widetilde{W}^{-}\right) \in \sigma_{\rho}$. Обозначим $\delta^{ \pm}=\widetilde{W}^{ \pm}-W^{ \pm}, \varepsilon^{ \pm}=\widetilde{F}^{ \pm}-F^{ \pm}$. Из (4.3) и из соответствующих соотношений для $\widetilde{W^{ \pm}}$получаем $\varepsilon^{ \pm}=P^{ \pm}\left(\widetilde{W^{+}} \delta^{-}+\delta^{+} W^{-}\right)$. Следовательно, $\left\|\left(\varepsilon^{+}, \varepsilon^{-}\right)\right\| \leqslant q\left\|\left(\delta^{+}, \delta^{-}\right)\right\|$.

Итак, в условиях леммы оператор $H$ является сжимающим в $\sigma_{\rho}$ и $(5.7)$ имеет единственное решение $\left(W^{+}, W^{-}\right)$в $\sigma_{\rho}$. Это решение является пределом следующих простых итераций:

$$
\left(W_{n+1}^{+}, W_{n+1}^{-}\right)=H\left(W_{n}^{+}, W_{n}^{-}\right), \quad W_{0}^{ \pm}=0, \quad n=0,1, \ldots
$$

Если $T^{ \pm} \geqslant O$, то последовательности $W_{n}^{ \pm} \geqslant O$ и их пределы $W^{ \pm}$также положительны. Лемма доказана.

5.4. Построение решения НУФ в случае $K \in \widetilde{B}$. Ниже будет изложен способ построения факторизации на основе леммы 5.2. Эта процедура будет разбита на следующие три этапа: построение базисного решения, продолжение решения и замыкание продолжения решения.

Обозначим через $\tilde{J}=\tilde{J}(K) \subset[a, b]$ следующее множество: $r \in \tilde{J}$, если при $s=r$ существует факторизация (5.6), где $\left(U_{r}^{+}, U_{r}^{-}\right) \in \widetilde{B}_{r}^{+} \times \widetilde{B}_{r}^{-}$, причем если $K \geqslant O$, то $U_{r}^{ \pm} \geqslant O$. Согласно лемме 5.1 при $r \in \tilde{J}$ существует факторизация (5.6) для любого $s \in[a, r]$, причем если $K \geqslant O$, то $U_{s}^{ \pm} \geqslant O$.

Обозначим $\tilde{\beta}=\sup \tilde{J} \leqslant b$. Опишем множество $\tilde{J}$. Пусть $K \in \widetilde{B}$. Тогда можно выбрать число $r>a$ такое, чтобы выполнялось неравенство $\left\|K_{a r}\right\|<\frac{1}{4}$. Рассмотрим уравнение $(5.7)$, где $T=K_{r}\left(=K_{a r}\right)$. Согласно лемме 5.2 это уравнение имеет решение $\left(U_{r}^{+}, U_{r}^{-}\right) \in \widetilde{B}_{r}^{+} \times \widetilde{B}_{r}^{-}$и имеет место факторизация $I-K_{r}=\left(I-U_{r}^{+}\right)\left(I-U_{r}^{-}\right)$, причем $\left(I-U_{r}^{ \pm}\right)^{-1}=I+V_{r}^{ \pm}, V_{r}^{ \pm} \in B_{r}^{ \pm}$.

Продолжим это решение. Пусть $r \in \tilde{J}$. Покажем, что тогда факторизация существует при значениях $s>r$, достаточно близких к $r$. При $s \in(r, b]$ имеем

$$
I-K_{s}=I-K_{r}-\left(K_{s}-K_{r}\right)=\left(I-U_{r}^{+}\right)\left(I-U_{r}^{-}\right)-K_{r s},
$$

откуда получаем

$$
I-K_{r}=\left(I-U_{r}^{+}\right)\left(I-T_{r s}\right)\left(I-U_{r}^{-}\right)
$$

где

$$
T_{r s}=\left(I+V_{r}^{+}\right) K_{r s}\left(I+V_{r}^{-}\right), \quad \mu=\left\|T_{r s}\right\| \leqslant\left(1+\left\|V_{r}^{+}\right\|\right)\left(1+\left\|V_{r}^{-}\right\|\right)\left\|K_{r s}\right\| .
$$

В силу $K \in \widetilde{B}$ можно выбрать $s>r$ такое, чтобы выполнялось неравенство $\mu<\frac{1}{4}$. Тогда согласно лемме 5.2 оператор $I-T_{r s}$ допускает факторизацию:

$$
I-T_{r s}=\left(I-U_{r s}^{+}\right)\left(I-U_{r s}^{-}\right),
$$

где $U_{r s}^{ \pm} \in B_{r s}^{ \pm},\left(I-U_{r s}^{ \pm}\right)^{-1}=I+V_{r s}^{ \pm}, V_{r s}^{ \pm} \in B_{r s}^{ \pm}$. 
Из (5.11) и (5.13) получаем $I-K_{s}=\left(I-U_{r}^{+}\right)\left(I-U_{r s}^{+}\right)\left(I-U_{r s}^{-}\right)\left(I-U_{r}^{-}\right)$. Учитывая (4.2), приходим к (5.6), где $U_{s}^{+}=U_{r}^{+}+U_{r s}^{+}, U_{s}^{-}=U_{r}^{-}+U_{r s}^{-}$. Если $K \geqslant O$, то $U_{s}^{ \pm} \geqslant O, V_{s}^{ \pm} \geqslant O$.

Поскольку построенные операторы $U_{s}^{ \pm}$дают факторизацию (5.6), то они являются решениями НУФ (5.4).

Нами доказана следующая лемма.

Лемма 5.3. Пусть $K \in \widetilde{B}$. Если $\tilde{\beta}=\sup \tilde{J}<b$, mo $\tilde{\beta} \notin \tilde{J} u \tilde{J}=[a, \tilde{\beta})$. Eсли $\tilde{\beta}=b$, то возможны два случая: $\tilde{J}=[a, b)$ и $\tilde{J}=[a, b]$.

5.5. Замыкание продолжения. При $r \in \tilde{J}$ имеем $\left(I-U_{r}^{ \pm}\right)^{-1}=I+V_{r}^{ \pm}$, $V_{r}^{ \pm} \in B_{r}^{ \pm}$.

Лемма 5.4. Справедливы утверждения:

i) если $[a, s) \subset \tilde{J}$ и операторы $V_{r}^{ \pm}, r \in[a, s)$, равномерно по $r$ ограничены по норме:

$$
\left\|V_{r}^{ \pm}\right\|_{B} \leqslant \alpha^{ \pm}
$$

то $s \in \tilde{J}$, m.е. существует факторизация (5.6);

ii) если $K \geqslant O$, то $U_{s}^{ \pm} \geqslant O, V_{s}^{ \pm} \geqslant O$.

ДокАЗАТЕЛЬСтво. Воспользуемся разложением (5.11). Из соотношений (5.12) и (5.14) получаем

$$
\mu=\left\|T_{r s}\right\| \leqslant\left(1+\alpha^{+}\right)\left(1+\alpha^{-}\right)\left\|K_{r s}\right\| .
$$

В силу $K \in \widetilde{B}$ можно выбрать $r<s$ такое, чтобы выполнялось неравенство $\mu<\frac{1}{4}$. Тогда согласно лемме 5.2 оператор $I-T_{r s}$ допускает факторизацию (5.13), откуда следует факторизация (5.6). Поскольку утверждение іi) очевидно, лемма доказана.

В следующем параграфе будут рассмотрены два случая, в которых удается получить равномерные оценки вида (5.14).

\section{§ 6. Факторизационные теоремы}

6.1. Случай положительного ядра. Пусть $K \in \widetilde{B}, K \geqslant O$ и $l \in J$ (см. п. 3.2), т. е. существует обратный оператор

$$
\left(I-K_{r}\right)^{-1}=I+\Gamma_{r}, \quad \Gamma_{r} \in \widetilde{B}_{r} \quad \forall r \in[a, l], \quad \Gamma_{r} \geqslant O .
$$

Покажем, что тогда существует факторизация (5.6). Предположим противное. Из леммы 5.3 следует, что $\tilde{\beta}=\sup \tilde{J} \notin \tilde{J}, \tilde{\beta} \leqslant l$. При $r<\tilde{\beta}$ имеем $I+$ $\Gamma_{r}=\left(I+V_{r}^{-}\right)\left(I+V_{r}^{+}\right), V_{r}^{ \pm} \geqslant O$, т. е. $V_{r}^{+}+V_{r}^{-}+V_{r}^{-} V_{r}^{+}=\Gamma_{r}, V_{r}^{ \pm} \geqslant O$. Отсюда и из (3.4) следуют неравенства $O \leqslant V_{r}^{ \pm} \leqslant \Gamma_{l}^{ \pm}:=P^{ \pm}\left(\Gamma_{l}\right)$. Поэтому имеют место неравенства (5.14), где $\alpha^{ \pm}=\left\|\Gamma_{l}^{ \pm}\right\|_{B}$. Согласно лемме 5.4 имеет место факторизация (5.6) при $s=l$. Полученное противоречие показывает, что факторизация (5.6) существует для произвольного $s \in[a, l]$.

С учетом утверждения iv) леммы 5.1 и формулы (3.2) мы приходим к следующей теореме. 
Теорема 6.1. Справедливы следующие утверждения:

i) пусть $K \in \widetilde{B}$ имеет положительное ядро; для существования факторизации (5.6), где $U_{s}^{ \pm} \in \widetilde{B}_{s}^{ \pm}$, необходимо и достаточно, чтобъ оператор $I-K_{r}$ был $\widetilde{B}$-обратим для произвольного $r \in[a, s]$;

ii) если при этом $\widetilde{B}$ является (хотя бы односторонним) идеалом в алгебре $\Omega$ линейных ограниченных операторов, действующих в $E$, то для существования факторизачии (5.6) с $U_{s}^{ \pm} \in \widetilde{B}_{s}^{ \pm}$необходимо и достаточно, чтобы при любом $r \in(a, s]$ уравнение

$$
f(x)=g(x)+\int_{a}^{r} k(x, t) f(t) \mu(d t), \quad x \in(a, r),
$$

было однозначно разрешимо в пространстве $E_{r}$ для любого $g \in E_{r}$.

Заметим, что в случае $s=b$ теорема 6.1 дает необходимое и достаточное условие существования факторизации (1.2) в случае положительного ядра.

6.2. Уравнения типа Гельфанда-Левитана. В случае интегральных операторов Гильберта-Шмидта связь между обратимостью операторов $I-K_{r}$ и существованием канонической факторизации (1.2) удается установить путем сочетания леммы 5.4 с возможностями уравнения типа Гельфанда-Левитана. В [1] изложен простой вывод этих уравнений в случае интегрального оператора Гильберта-Шмидта вида (1.5).

Пусть $K \in B$ и имеет место каноническая факторизация (5.6). Методом, использованным в [1], покажем, что ядра $\nu^{ \pm}=\nu_{s}^{ \pm}$вольтерровых операторов $V_{s}^{ \pm}$(определяемых согласно (3.1)) удовлетворяют следующим линейным уравнениям типа Гельфанда-Левитана:

$$
\begin{array}{ll}
\nu^{+}(x, y)=k(x, y)+\int_{a}^{x} \nu^{+}(x, t) k(t, y) \mu(d t), & a<y<x<s, \\
\nu^{-}(x, y)=k(x, y)+\int_{a}^{y} k(x, t) \nu^{-}(t, y) \mu(d t), & a<x<y<s .
\end{array}
$$

Зависимость функций $\nu^{ \pm}$от $s$ выражается только зависимостью от $s$ областей определения этих функций.

Из (1.2) и (3.1) имеем $V_{s}^{+}=K_{s}+U_{s}^{+}+V_{s}^{+} K_{s}$. Применяя к этому равенству проектор $P^{+}$, с учетом $P^{+}\left(U_{s}^{-}\right)=0$ получаем уравнение $V_{s}^{+}=K_{s}^{+}+P^{+}\left(V_{s}^{+} K_{s}\right)$. Аналогичным образом получается уравнение $V_{s}^{-}=K_{s}^{-}+P^{-}\left(K_{s} V_{s}^{-}\right)$. Здесь, как и раньше, $K_{s}^{ \pm}=P^{ \pm}\left(K_{s}\right), K_{s}=Q_{s}(K)$. Перейдя в этих уравнениях к ядрам, приходим к уравнениям (6.2).

Отметим то обстоятельство, что уравнения (6.2) выведены при условии существования факторизации (1.2), что не гарантирует их эквивалентность задаче (1.2). Сказанное в равной степени относится к классическим уравнениям Гельфанда-Левитана.

Пусть существует резольвента $\Gamma_{r}$ оператора $K_{r}$ для произвольного $r \leqslant s$. Обозначим через $\gamma(x, t, r)$ резольвентное ядро. Решая через резольвенты уравнения (6.4) и (6.5) при фиксированных $x$ и $y$ соответственно, получаем следующие формулы для $\nu^{ \pm}=\nu_{s}^{ \pm}$:

$$
\begin{array}{ll}
\nu^{+}(x, y)=\nu_{s}^{+}(x, y)=k(x, y)+\int_{a}^{x} k(x, t) \gamma(t, y, x) \mu(d t), & a<y<x<s, \\
\nu^{-}(x, y)=\nu_{s}^{-}(x, y)=k(x, y)+\int_{a}^{y} \gamma(x, t, y) k(t, y) \mu(d t), & a<x<y<s .
\end{array}
$$


Сложность оценивания норм функций $\nu_{s}^{ \pm}$с помощью формул (6.3) связана с тем, что под интегралами оба аргумента ядра $k$ являются также аргументами резольвенты $\gamma$.

6.3. Случай оператора Гильберта-Шмидта. Рассмотрим теперь тот случай, когда исходным пространством $E$ служит $L_{2}(\mu)$, а пространством $B$ служит класс $H_{2}(\mu)$ интегральных операторов Гильберта-Шмидта с нормой (2.6). Для этого класса, очевидно, выполняются условия (2.16). Поэтому в данном случае пространства $B, \widetilde{B}, \breve{B}$ совпадают.

Обозначим через $\mu_{s}$ следующую меру: $\mu_{s}(d x)=\kappa_{s} \mu(d x)$, где, как и раньше, $\kappa_{s}$ - характеристическая функция интервала $(a, s)$.

В рассматриваемом случае исходного пространства $E=L_{2}(\mu)$ пространством $E_{s}$ служит $L_{2}\left(\mu_{s}\right)$.

Предположим, что при некотором $r \in(a, b]$ уравнение (6.1) разрешимо в пространстве $L_{2}\left(\mu_{r}\right)$ для любого $g \in L_{2}\left(\mu_{r}\right)$, т. е. существует оператор $\left(I-K_{r}\right)^{-1}=I+\Gamma_{r} \in \Omega$. Поскольку $H_{2}(\mu)$ является (двусторонним) идеалом в $\Omega$, то $\Gamma_{s} K_{r} \in \widetilde{B}_{r}$, поэтому $\Gamma_{r}=K_{r}+\Gamma_{r} K_{r} \in \widetilde{B}_{r}$, т. е.

$$
\left(I-K_{r}\right)^{-1}=I+\Gamma_{r}, \quad \Gamma_{r} \in H_{2}\left(\mu_{r}\right) .
$$

Пусть оператор $I-K_{r}$ обратим для произвольного $r \leqslant s$. Тогда согласно утверждению iii) леммы 3.1 имеет место равномерная по $r$ оценка

$$
\varphi^{2}(r)=\left\|\Gamma_{r}\right\|_{B}^{2}=\iint \gamma^{2}(x, t, r) \mu_{r}(d x) \mu_{r}(d t) \leqslant c_{s}^{2}<+\infty \quad \forall r \leqslant s, \quad c_{s}>0 .
$$

Ниже с помощью формул (6.3) будут получены оценки для норм ядер $\nu_{r}^{ \pm}$операторов $V_{r}^{ \pm}$.

Лемма 6.1. Пусть оператор $I-K_{r}$ обладает обратным для произвольного $r \leqslant s$ и факторизачия (5.6) существует при $r<s$. Тогда имеют место следующие равномерные по $r$ оценки:

$$
\left\|\nu_{r}^{ \pm}\right\|_{B} \leqslant\|k\|_{B}\left(1+c_{s}\right), \quad c_{s}>0 .
$$

ДокАзАТЕЛЬство. Используя неравенство Коши-Буняковского, получаем

$$
\left|\int_{a}^{x} k(x, t) \gamma(t, y, x) \mu(d t)\right|^{2} \leqslant \int_{a}^{x} k^{2}(x, t) \mu(d t) \int_{a}^{x} \gamma^{2}(t, y, x) \mu(d t) .
$$

Интегрируя это неравенство по мере $\mu(d y)$ от $a$ до $x$, с учетом неравенства (6.5) получаем

$$
\int_{a}^{x}\left|\int_{a}^{x} k(x, t) \gamma(t, y, x) \mu(d t)\right|^{2} \mu(d y) \leqslant c_{s}^{2} \int_{a}^{x} k^{2}(x, t) \mu(d t), \quad x \leqslant s,
$$

откуда имеем

$$
\int_{a}^{r} \mu(d t) \int_{a}^{x}\left|\int_{a}^{x} k(x, t) \gamma(t, y, x) \mu(d t)\right|^{2} \mu(d y) \leqslant c_{s}^{2}\left\|K_{r}\right\|_{B}^{2} \leqslant c_{s}^{2}\left\|K_{s}\right\|_{B}^{2}, \quad r<s .
$$

Из формул (6.3) и (6.7) следует первая оценка из (6.6). Вторая оценка из формулы (6.6) получается аналогичным образом. Лемма доказана. 
Из лемм 5.4, 6.1 и утверждения iv) леммы 5.1 следует

Теорема 6.2. Пусть $K \in H_{2}(\mu)$ - интегральный оператор ГильбертаШмидта вида (1.1), где $\mu$ - мера, непрерывная относительно меры Лебега. Для существования факторизачии (1.4), где $V^{ \pm}$- вольтерровы операторы из $H_{2}(\mu)$ вида (1.3), необходимо и достаточно, чтобы при любом $r \in(a, b]$ уравнение (6.1) было разрешимо в пространстве $L_{2}\left(\mu_{r}\right)$ для любого $g \in L_{2}\left(\mu_{r}\right)$.

\section{Список литературы}

1. И.Ц. Гохберг, М.Г. Крейн, Теория волътерровых операторов в гильбертовом пространстве и ее приложения, Наука, М., 1967; англ. пер.: I. C. Gohberg, M. G. Krein, Theory and applications of Volterra operators in Hilbert space, Transl. Math. Monogr., 24, Amer. Math. Soc., Providence, RI, 1970.

2. Н.Б. Енгибарян, "Нестационарная диффузия излучения", Астрофизика, 2:2 (1966), 197-204.

3. H. H. Kagiwada, R. E. Kalaba, A. Schumitzky, "An initial-value method for Fredholm integral equations", J. Math. Anal. Appl., 19:1 (1967), 197-203.

4. Н. Б. Енгибарян, "О факторизации симметрических интегральных операторов", Докл. АН СССР, 203:1 (1972), 19-21; англ. пер.: N. B. Engibaryan, "On the factorization of symmetric integral operators", Soviet Math. Dokl., 13:1 (1972), 322-325.

5. Н. Б. Енгибарян, "О нелинейных уравнениях факторизации операторов", Применение методов теории функиий и функи. анализа к задачам мат. физики, Изд-во ЕрГУ, Ереван, 1982, 123-128.

6. Н. Б. Енгибарян, "Некоторые факторизационные теоремы для интегральных операторов”, Докл. АН СССР, 230:5 (1976), 1021-1024; англ. пер.: N. B. Engibaryan, "Some factorization theorems for integral operators", Soviet Math. Dokl., 17:5 (1976), $1418-1422$.

7. Л. Г. Арабаджян, Н. Б. Енгибарян, "Уравнения в свертках и нелинейные функциональные уравнения”, Итоги науки и техн. Сер. Матем. анал., 22, ВИНИТИ, M., 1984, 175-244; англ. пер.: L. G. Arabadzhyan, N. B. Engibaryan, "Convolution equations and nonlinear functional equations", J. Math. Sci., 36:6 (1987), 745-791.

8. Н.Б. Енгибарян, "Постановка и решение некоторых задач факторизации интегральных операторов", Матем. сб., 191:12 (2000), 61-76; англ. пер.: N. B. Engibaryan, "Setting and solving several factorization problems for integral operators", $S b$. Math., 191:12 (2000), 1809-1825.

9. N. B. Yengibarian, "Factorization of Markov chains", J. Theoret. Probab., 17:2 (2004), 459-481.

10. М. А. Красносельский, П. П. Забрейко, Е. И. Пустыльник, П. Е. Соболевский, Интегральные операторы в пространствах суммируемых функиий, Наука, М., 1966; англ. пер.: М. А. Krasnosel'skij, P.P. Zabrejko, E. I. Pustylnik, P. E. Sobolevskij, Integral operators in spaces of summable functions, Noordhoff, Leyden, 1976.

Н. Б. ЕНГИБАрян (N. B. Yengibaryan)

Поступило в редакцию

Институт математики НАН Армении

02.08.2007

E-mail: yengib@instmath.sci.am 